\title{
Identification of a circuit-based endophenotype for familial depression
}

\author{
Marc Dubin $^{\mathrm{a}, \mathrm{c}, *}$, Myrna Weissman ${ }^{\mathrm{b}, \mathrm{c}}$, Dongrong Xu ${ }^{\mathrm{a}, \mathrm{c}}$, Ravi Bansal ${ }^{\mathrm{a}, \mathrm{c}}$, Xuejun Hao ${ }^{\mathrm{a}, \mathrm{c}}$, Jun Liu ${ }^{\mathrm{a}, \mathrm{c}}$, \\ Virginia Warner $^{\mathrm{b}}$, Bradley Peterson ${ }^{\mathrm{a}, \mathrm{c}}$ \\ a The Division of Child and Adolescent Psychiatry, The New York State Psychiatric Institute, 1051 Riverside Drive, New York, NY 10032, USA \\ b The Division of Epidemiology, College of Physicians and Surgeons, Columbia University, New York, NY, USA \\ ' The Department of Psychiatry, College of Physicians and Surgeons, Columbia University, New York, NY, USA
}

\section{A R T I C L E I N F O}

\section{Article history:}

Received 15 April 2011

Received in revised form 14 September 2011

Accepted 21 November 2011

\section{Keywords:}

Unipolar depression

Family study

Endophenotype

Biological markers

Structural neuroimaging

Brain imaging techniques

\begin{abstract}
A B S T R A C T
Frontal and parietal lesions may cause depression, and cortical thinning of the right frontal and parietal lobes has been shown to be a marker of risk for familial major depression. We studied biological offspring within a three-generation cohort, in which risk was defined by the depression status of the first generation, to identify regional volume differences associated with risk for depression throughout the cerebrum. We found reduced frontal and parietal white matter volumes in the high-risk group, including in persons without any personal history of depression, suggesting that hypoplasia of frontal and parietal white matter is an endophenotype for familial depression. In addition, white matter volumes in these regions correlated with current severity of symptoms of depression, inattention, and impulsivity. White matter volumes also correlated strongly with the degree of thinning in the right parietal cortex. These findings support a model of pathogenesis in which hypoplasia within a neural network for attention and emotional processing predisposes to depression.

(c) 2012 Elsevier Ireland Ltd. All rights reserved.
\end{abstract}

\section{Introduction}

Environmental and biological factors determine an individual's risk of developing major depressive disorder (Hill et al., 2004). Adverse events in early life, early adverse environmental factors, and genetic loading conspire to predispose to the illness. Having a first degree relative with depression elevates one's risk of depression several-fold (Gershon, 1983). Monozygotic twins have a much greater rate of concordance (69\%) than dizygotic twins (McGuffin, 1984), independent of being raised together or apart (Price, 1968). Familial depression may be associated with lower rates of suppression in the dexamethasone suppression test (Mendlewicz et al., 1982).

Identifying an endophenotype of risk for psychiatric illness offers the possibility of predicting the development of illness in susceptible individuals, which in turn could lead to early treatment and prevention and to the ability to predict a response to specific treatments (Cook et al., 2009). Here, we adopted the definition of endophenotype as a biomarker that is associated with the illness in the population, is heritable, is state-independent, and co-segregates with the illness within families (Gottesman and Gould, 2003). Endophenotypes can also provide important clues to the causes of psychiatric illness, which can, in turn, provide insight into better methods of early diagnosis, prevention, and treatment.

\footnotetext{
* Corresponding author at: 525 East 68th St., Box 140, New York, NY, 10065, USA. Tel.: +1212746 5817; fax: + 12127468800 .

E-mail address: mrd9035@med.cornell.edu (M. Dubin).
}

We recently reported a robust endophenotype of risk for major depressive disorder (MDD) (Peterson et al., 2009). We found that persons at high risk for major depression, based on their family history, have thinner right frontal, parietal, posterior temporal, and occipital cortices than do low-risk individuals. This endophenotype was present even in high-risk individuals who had no history of MDD, thus indicating that the thinning was not a consequence of the illness or its treatment. As our previous study was restricted to assessing the anatomical features of the cortical mantle, we now sought structural endophenotypes in the cerebrum deep down to the cortex. Therefore, we measured local volumes throughout the cerebrum and assessed their associations with an individual's familial risk status for MDD. We assessed the degree of interdependence of subcortical volumes and cortical thinning to determine whether subcortical volumetric and cortical thinning endophenotypes each accounted for unique portions of the variance of risk. As the findings of the current study were extensive, we decided to present them independent from the previously published findings on cortical thinning.

Prior studies have reported both anatomical and functional abnormalities in the frontal lobes of depressed individuals. Stroke- and trauma-induced damage to the frontal lobes can cause depression (Robinson and Price, 1982; Jorge et al., 2004). Idiopathic depression is associated with volume loss in the dorsolateral prefrontal cortex, amygdala, and hippocampus (Frodl et al., 2008; Amico et al., 2011), and these markers interact with early childhood stress to increase the risk of a more severe course of illness (Frodl et al., 2010). Familial depression is associated with reduced glucose metabolism in the subgenual cingulate cortex (Drevets et al., 1997). Depression is also 
Table 1

Pairwise correlation coefficients between average thicknesses of cortical areas.

\begin{tabular}{lll}
\hline & $\begin{array}{l}\text { Correlation between right } \\
\text { hemispheric thicknesses }\end{array}$ & $\begin{array}{l}\text { Correlation between left } \\
\text { hemispheric thicknesses }\end{array}$ \\
\hline Middle frontal-precentral & 0.84 & 0.82 \\
$\begin{array}{c}\text { Postcentral-inferior } \\
\text { parietal }\end{array}$ & 0.89 & 0.81 \\
$\begin{array}{c}\text { Postcentral-middle } \\
\text { temporal }\end{array}$ & 0.83 & 0.76 \\
$\begin{array}{c}\text { Middle temporal-inferior } \\
\text { parietal }\end{array}$ & 0.84 & 0.63 \\
\hline
\end{tabular}

Pairwise correlation coefficients between average thickness of two frontal cortical areas (middle frontal gyrus and precentral gyrus) and three parietal and temporal cortical areas (postcentral gyrus, inferior parietal lobule, and middle temporal gyrus). Correlation equations are written as dependent variable-independent variable. Correlation coefficients are shown between the two frontal areas and between the three parietal areas and are shown for both the right and left hemispheres.

associated with abnormal reward processing and altered activation of the frontal cortices (Tremblay et al., 2005). Treatment of depression with either psychotherapy or medication alters metabolism of the frontal cortices (Seminowicz et al., 2004). These findings, coupled with our prior finding that thinning of frontal and parietal cortices predisposes to the development of MDD, led us to hypothesize in the present study that we would detect reduced volume of the white matter of the frontal and parietal lobes in familial MDD.

\section{Materials and methods}

\subsection{Subjects}

This study was initiated in 1982 and the full details of design, sample selection, and assessments of the three generations have been reported (Weissman et al., 2005). The participants included 131 individuals in a longitudinal study of family members at high risk for major depression. Members of the first generation (G1) who had a history of MDD or no history of MDD were recruited, as were their children (G2) and grandchildren (G3). Risk status was defined as the clinical status of G1 (e.g. G2 and G3 were high risk if G1 had a history of MDD and G2 and G3 were low risk otherwise) and risk status did not change when a G2 or G3 member developed a depressive episode. High-risk and low-risk samples were drawn from the same community. Participants in all three generations have been followed over the past 27 years, with clinical assessments at 5-year intervals or "waves". Thus far, five waves have been completed. All imaging data were obtained in wave 5 . All assessment and imaging procedures were undertaken with Institutional Review Board (IRB) approval of both Yale and the New York State Psychiatric Institute. Written consent was obtained for all participants over 18 years old and assent was obtained for participants 17 years or younger. Exclusion criteria were current psychotic symptoms (that would interfere with the consent process), current pregnancy, and ferromagnetic implants.

From the $91 \mathrm{G} 1$ families in the study, $131 \mathrm{G} 2$ and G3 participants from 49 distinct families were scanned. Data from this same set of 131 scans were presented in the previous article (Peterson et al., 2009). Of these 131 G2 and G3 participants, 66 individuals constituted the high-risk group ( 12 children, defined as persons younger than 18 years of age, and 54 adults, 35 females, 54 right-handed; mean age 33.3 years [standard deviation (S.D.) 12.9], range 6.3-54.5 years) and 65 individuals made up the low-risk group ( 31 children, 34 adults; 35 females; 54 right-handed; mean age 24.8 years [S.D. 13.1], range 7.9-49.5 years). The average age in the high-risk group was significantly higher than in the low risk $(p<0.01)$ and thus age was used as a covariate in all linear models. All participants were Caucasian.

The primary difference between the previous report (Peterson et al., 2009) and the present one is the anatomical region of study and the measures of volumetric difference. We investigate volumetric differences throughout both cerebral hemispheres, whereas the previous report focused on differences in thickness within the cerebral cortex.

\subsection{Procedures}

\subsubsection{Clinical assessments}

Participants were diagnosed using a semi-structured diagnostic interview (the Schedule for Affective Disorders and Schizophrenia - Lifetime Version for adults and the child version of the instrument that was modified for the Diagnostic and Statistical Manual of Mental Disorders, edition 4 [DSM-IV] for participants 6-17 years of age). Diagnostic interviews were performed by doctoral and master's level interviewers and best-estimate diagnosis was determined by an independent review by a child psychiatrist and child psychologist who were blind to the participant's risk group. Agreement between evaluators was good to excellent as determined by interrater Cohen's kappa coefficients (two clinicians) at wave 4 of 178 randomly selected participants from all generations. MDD: 0.82, dysthymia, 0.89; anxiety disorder, 0.65; alcohol abuse/dependency, 0.94; and drug abuse/dependency, 1.00 .

The Hamilton Depression Rating Scale was used to measure depressive symptoms in adults and the Children's Depression Rating Scale-Revised was used to assess these symptoms in children. The Hamilton Anxiety Rating Scale inventory was used to measure anxiety symptoms in adults and the Revised Children's Manifest Anxiety Scale was used in children. An index of the severity of depressive or anxiety symptoms across children and adults was computed by calculating a $z$-score for each participant, and those $z$-scores then were combined across age groups into a single variable for each symptom domain, i.e., "z-depression," " $z$-anxiety," or " $z$-anxiety/depression (zanxdep)" (the sum of $z$-depression and $z$-anxiety scores). The resulting $z$-scores were thus normalized according to age group and were used in correlation analyses. Because individuals who suffer from major depression also have difficulty with inattention, and because attention deficit hyperactivity disorder (ADHD) is often comorbid with MDD in adolescents (Faraone and Biederman, 1997), we used the DuPaul-Barkley ADHD rating scale at the time of scanning in wave 5 to measure the symptoms of inattention, hyperactivity, and impulsivity.

\subsubsection{Magnetic resonance imaging (MRI) scanning}

High-resolution MRI scans were obtained using a Siemens Sonata $1.5 \mathrm{~T}$ scanner (Siemens AG) equipped with high-speed gradients (maximum amplification, $40 \mathrm{mT} / \mathrm{m}$; slew rate, $200 \mathrm{mT} / \mathrm{m} / \mathrm{s}$ ). Head positioning was standardized using canthomeatal landmarks. Brain scans were acquired using a 3D Magnetization-Prepared Rapid Acquisition with Gradient Echo (MP-RAGE) sequence (repetition time, $24 \mathrm{~ms}$; echo time, $2.96 \mathrm{~ms}$; $45^{\circ}$ flip angle; $256 \times 192$ matrix; field of view, $30 \mathrm{~cm}$; phase field of view $=100 \%$; two excitations, slice thickness, $1.2 \mathrm{~mm}$; and 124 contiguous slices encoded for sagittal slice reconstruction with voxel dimensions of $1.17 \times 1.17 \times 1.2 \mathrm{~mm}$ ).

Large-scale variations in image intensity were corrected using a validated algorithm developed at the Montreal Neurological Institute (Sled et al., 1998). Extracerebral tissues were removed using an automated tool for extracting the brain that smooths image intensity using an anisotropic filter, detects 3D edges using a Marr-Hildreth edge detector, and then defines the brain as the largest connected component with a closed boundary (Smith, 2002). Connecting dura was removed manually on each slice in the sagittal view and confirmed in the orthogonal views. The brainstem was transected at the pontomedullary junction.

\subsubsection{Image analysis}

Using the deformation field that normalizes a brain into the template space in surface analyses, we first applied volume preserved warping (VPW) to transform a binarized image of the brain to generate a spatially normalized image with varying pixel intensities. In this normalized image, regions of high intensity denote regions compressed by the deformation field, and regions of low intensity denote regions that were expanded by the deformation field. Thus regions of high VPW intensity are those that have higher volume in the participant's brain than in the template brain, and regions of low VPW intensity are those that have lower volume (or "hypoplasia," a term used throughout this article, consistent with our hypothesis that the lower volumes reflect brain underdevelopment) in the participant's brain relative to the template brain. We then perform voxel-wise statistical analyses to test hypotheses that risk group status, current symptoms of depression or anxiety, and combined symptoms of ADHD correlate with VPW measures. These methods have been described extensively elsewhere and were implemented using software developed by Dr. Dongrong Xu at Columbia University (Xu et al., 2007).

\subsubsection{Statistical analysis}

We tested the hypothesis that VPW measures in the frontal and parietal white matter would be smaller in the high-risk than low-risk group by conducting a voxelwise comparison of risk groups using a linear regression with VPW as the dependent variable, risk group as the independent variable, and age and sex as covariates. We studied the relationship between VPW and current symptom severity (zanxdep), using a linear model with dependent variable VPW, independent variable zanxdep and age, sex and risk group as covariates. We chose zanxdep as we observed similar effects when we considered $z$-anxiety and $z$-depression separately. The relationship between VPW and combined and component ADHD scores was assessed similarly. We then performed each correlation analysis on each risk group separately to exclude the possibility that significant correlations were driven by differences in the independent variable between the two risk groups. $P$-values were color-coded and plotted for each voxel in the volume of the cerebral hemispheres. False discovery rate (FDR) with a threshold of $10 \%$ was used to correct for multiple comparisons (Benjamini and Hochberg, 1995). This corresponds to the estimate that $10 \%$ of the color-coded pixels are false positive statistical tests. We used two complementary approaches to assess the interdependence of our VPW findings from our prior finding of reduced cortical thickness in this sample. In one approach, VPW was used as the dependent variable in a regression analysis that correlated VPW measures at each voxel of the brain with the average cortical thickness over a given cortical region while covarying for age, sex, and risk group. Because of the large expanse of cortical thinning previously reported, we selected representative cortical regions from frontal, parietal, and temporal cortices. The cortical regions were selected from the University of California at Los Angeles (UCLA) probabilistic atlas (Dinov et al., 2000; Mazziotta et al., 2001). In a second approach, cortical thickness at each voxel on the cortical surface was used as the dependent variable in a regression that correlated thickness with average VPW over 
the region of significant effect of risk group, while covering for age, sex, and risk status. In each case, the model was initially run while including the interaction effects of riskby-VPW or risk-by-thickness. If the interaction effect was not statistically significant at a given voxel, the model was re-run without the interaction term.

\section{Results}

\subsection{Clinical measures}

The data set consisted of 131 individuals; 66 (12 children and 54 adults) were in the high-risk group and 65 (31 children and 34 adults) in the low-risk group. As expected, the frequency of lifetime MDD was significantly greater in the high-risk group $(n=37,57 \%)$ than in the low-risk group $(n=15,23 \%)\left(\chi^{2}=13.54\right.$, degree of freedom (d.f.) $=1, p<0.001$ ), as was the frequency of lifetime anxiety disorder (high-risk: $n=34,52 \%$; low-risk: $n=14,21 \% ; \chi^{2}=11.42$, d.f. $=1, p<0.001$ ) (Supplemental Table 1). These rates of lifetime MDD and anxiety disorder in the low-risk group were comparable to those reported in community surveys (Beidel and Turner, 1997; Wickramaratne and Weissman, 1998). The high-risk group compared with the low-risk group had significantly more MDD (high-risk: $n=16,25 \%$; low-risk: $n=7,11 \% ; \chi^{2}=4.28$, d.f. $=1, p<0.05$ ) but not anxiety disorder (high-risk group: $n=23,35 \%$; low-risk group: $n=16,25 \%$; chisq $=1.79$, d.f. $=1, p=0.18$ ) at the time of MRI scanning, and the groups were similar in the severity ratings of depression and anxiety symptoms (Hamilton Depression Rating Scale score in high-risk adults $=5.2$ [S.D. 6.1], range $=0-26$; in low-risk adults $=4.6$ [S.D. 6.0], range $=0-21 ; t=0.41$, d.f. $=80, p=0.68$; Hamilton Anxiety Rating Scale score in high-risk adults $=5.0$ [S.D. 5.4], range $=0-21$; in low-risk adults $=5.1$ [S.D. 7.3], range $=0-27$; $t=0.04$, d.f. $=80, p=0.97$ ).

\subsection{Relationship between clinical measures and white matter volume}

Local volumes in the high-risk group were reduced bilaterally in the white matter of the frontal and parietal lobes, internal capsule, and cerebral peduncle, confirming our hypothesis. These findings were present after correcting for scaling effects within the brain, and they persisted when covarying for age and sex. The local volume reduction in white matter of the right frontal and parietal lobes survived correction for multiple comparisons using the false discovery rate (FDR) (Fig. 1a). Local volumes were reduced in the subset of participants who had no lifetime history of MDD (Fig. 1b). Local volumes were reduced in children and adults considered separately and using age as a covariate within each group (Supplementary materials). No region was found to be larger in the high-risk group.

White matter volumes in the frontal and parietal lobes correlated inversely with the severity of current symptoms of depression and anxiety in the same regions as the effect of risk status (Fig. 2a). Similar effects were present when depression and anxiety symptoms were considered separately. Combined ADHD symptom severity also correlated inversely with white matter volume in the right frontal white matter, an effect that persisted when symptom sub-scores for impulsivity and inattention were considered separately (Fig. 2b). The correlations with symptom severity were observed for participants in the high- and low-risk groups combined (Fig. 2b) and in the high- and low-risk groups separately, indicating that these correlations likely hold for the general population, and not only for persons at high familial risk for depression. Scores on the Global Assessment Scale (GAS) correlated with white matter volumes in the bilateral frontal and parietal white matter (Fig. 2c), an effect that persisted after controlling for the severity of current symptoms. The correlation with GAS scores was stronger in the low-risk group than in the highrisk group, where limited variance and floor effects on local volumes interfered with our ability to detect significant correlations (not shown).

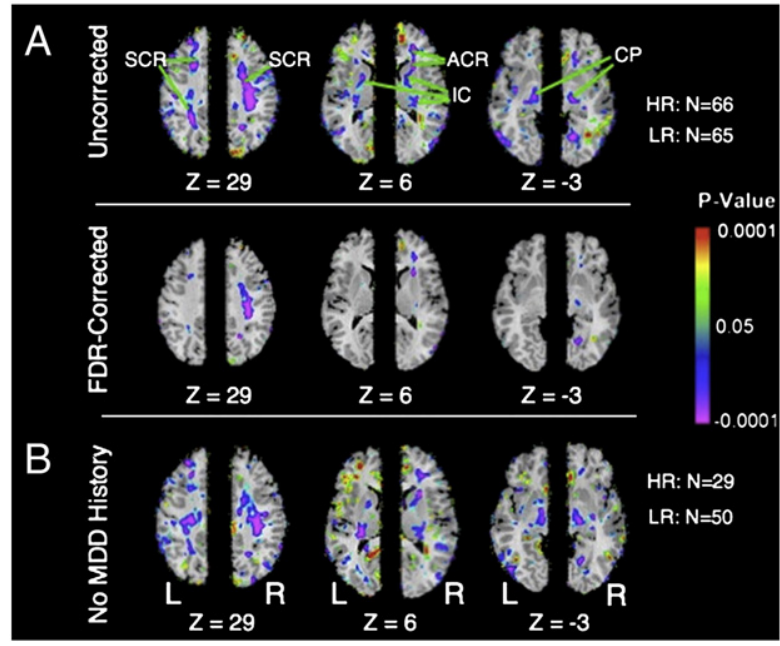

Fig. 1. Correlation of white matter volume and risk status. a. Maps of regional cerebral volume difference (VPW) between high- and low-risk groups. VPW is rescaled for the age and sex of all participants. Colors indicate voxels in which volume differences were significant $(p<0.05)$. Warm colors (yellow, orange, and red) represent greater local volume (hyperplasia) in the high-risk group; cooler colors (blue and purple) represent lesser local volume (hypoplasia) in that group. The color bar indicates the color-coding of $p$-values for testing of statistical significance at each voxel throughout the brain volume. In the upper row, $p$-values are thresholded at $p<0.05$ without correction for multiple comparisons. In the lower row, $p$-values are thresholded to control the false discovery rate (FDR) to $10 \%$ to correct for multiple comparisons. Risk correlates with white matter hypoplasia in the frontal and parietal lobes bilaterally. Three axial levels (Talairach $Z=29, Z=6$, and $Z=-3$ ) are shown. Volumes of white matter in the frontal and parietal lobes and cerebral peduncle are reduced in the high-risk group, suggesting that white matter hypoplasia in this region is an endophenotype of risk of MDD. SCR, superior corona radiata; ACR, anterior corona radiata; IC, internal capsule; $\mathrm{CP}$, cerebral peduncle; HR, high-risk; LR, low-risk; $Z$, Talairach $z$ coordinate. b. Maps of group differences in regional cerebral volume (VPW) between high- and low-risk groups in which no member had a lifetime history of MDD. Risk correlates with white matter hypoplasia in the frontal and parietal lobes bilaterally, independent of MDD history. Format as in (a).

\subsection{Relationship of cortical thickness with white matter VPW measures}

Average cortical thickness in Regions of interest (ROIs) within parietal and temporal cortices correlated positively and strongly $\left(r s>0.56, p s<10^{-6}\right)$ with VPW measures in the right and left superior corona radiata, internal capsule, and cerebral peduncle (Fig. 3, a-c). These correlations were much weaker ( $r s>0.38, p s<10^{-4}$ ) in the middle frontal and precentral gyri (Fig. 3, d,e).

Conversely, right hemisphere VPW measures, averaged over voxels where the effect of risk group was statistically significant, correlated with cortical thickness measures in the posterior lateral right hemisphere (Fig. 4a). The interaction of VPW $\times$ group was significant over the right posterior lateral convexity (Fig. 4b). Scatterplots illustrated that the interaction derived from a flattened slope in the high-risk group, which in turn was caused by reduced variances in both cortical thickness and VPW measures in the high-risk group (Fig. 4c). The distribution of VPW measures in the high-risk group was narrower than in the low-risk group (Fig. 4d) (high-risk: mean $=0.90, \quad$ S.D. $=0.006 ; \quad$ low-risk: mean $=0.96$, S.D. $=0.009$ $\left.F_{61,59}=1.65, p<0.06\right)$.

\section{Discussion}

We have identified a putative endophenotype for risk of MDD that consists of bilateral white matter hypoplasia in the frontal and parietal lobes and cerebral peduncles. This marker confirms our hypothesis that anatomical abnormalities in the frontal lobes contribute to the vulnerability to developing MDD, and only in part to developing symptoms of major depression. The marker also satisfies the criteria that define an endophenotype (Gottesman and Gould, 2003), in that 


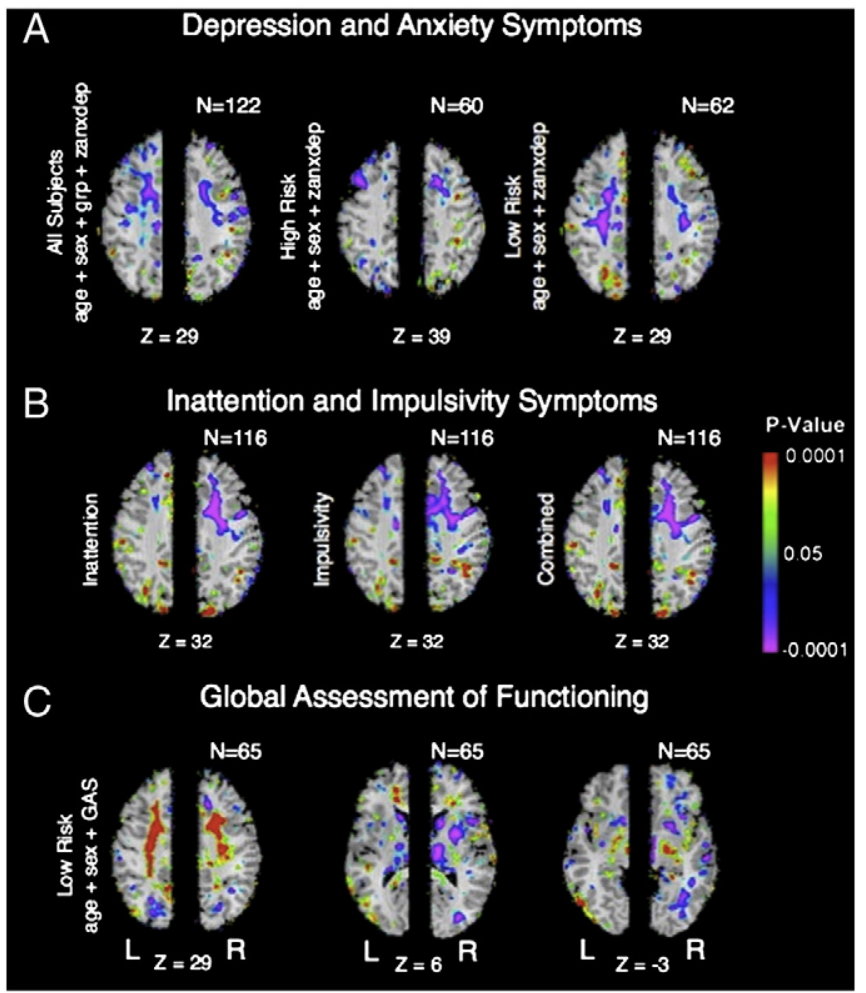

Fig. 2. Correlation of current symptoms and white matter volume. a. Correlations of VPW with current symptom severity. (Left) Correlation of VPW with $z$-anxdep, the sum of the $z$-depression and $z$-anxiety measures, in all participants, covarying for age, sex, and risk status. Colors indicate voxels in which volume differences correlated significantly with symptom severity $(p<0.05)$. Warm colors (yellow, orange, and red) represent significant positive correlations. Cooler colors (blue and purple) represent inverse correlations between VPW and symptom severity. Correlations of VPW with $z$-anxdep are also shown for the high-risk group only (middle) and low-risk group only (right). In the combined group and the low-risk group, frontoparietal white matter correlates negatively with $z$-anxdep. The region of significant correlation overlaps with the region of white matter hypoplasia (Fig. 1), indicating that white matter hypoplasia is present in direct proportion to the current severity of symptoms. Axial slices are shown for the $Z=29$ level except for the high-risk group in which the axial slice $Z=39$ illustrates the most significant correlation for this group. b. Correlations of VPW with the symptoms of ADHD (Talairach level $Z=32$ ). Correlations are shown for high-risk and low-risk groups combined, although similar maps were found in both the high-risk and low-risk groups separately. (Left) Inattention, (middle) impulsivity, and (right) DuPaul-Barkley score of combined inattention and impulsivity. The severity of current symptoms of ADHD correlates proportionately with the magnitude of white matter hypoplasia, a correlation that is strongest in the frontal and parietal white matter of the right hemisphere. Statistical models for the correlations include age and sex as covariates. $Z$, Talairach $z$-coordinate. c. Correlations of VPW with global assessment of functioning (Talairach levels $Z=29, Z=6, Z=-3$ ). Correlation of VPW measures with Global Assessment Scale (GAS) scores in low-risk participants while covarying for age and sex.

it is associated with the risk of developing the illness and is not simply a sequela of illness. White matter hypoplasia was relatively stateindependent and was detected in never-ill family members of the high-risk sample compared with members of the low-risk group. The degree of white matter hypoplasia correlated with current mood and anxiety symptoms and with current combined symptoms of ADHD in both the high- and low-risk groups separately, indicating that relative hypoplasia of white matter produces a proportionately greater burden of symptoms, regardless of familial risk status. These correlations with symptom severities were present even in those who have never been ill with MDD or an anxiety disorder, indicating that they confer a functional burden even in the absence of a diagnosable mood disorder. The correlations with symptom severities were limited to the right hemisphere, however, and they involved a smaller extent of white matter than did the association with familial risk, suggesting that white matter hypoplasia is more prominently a marker of

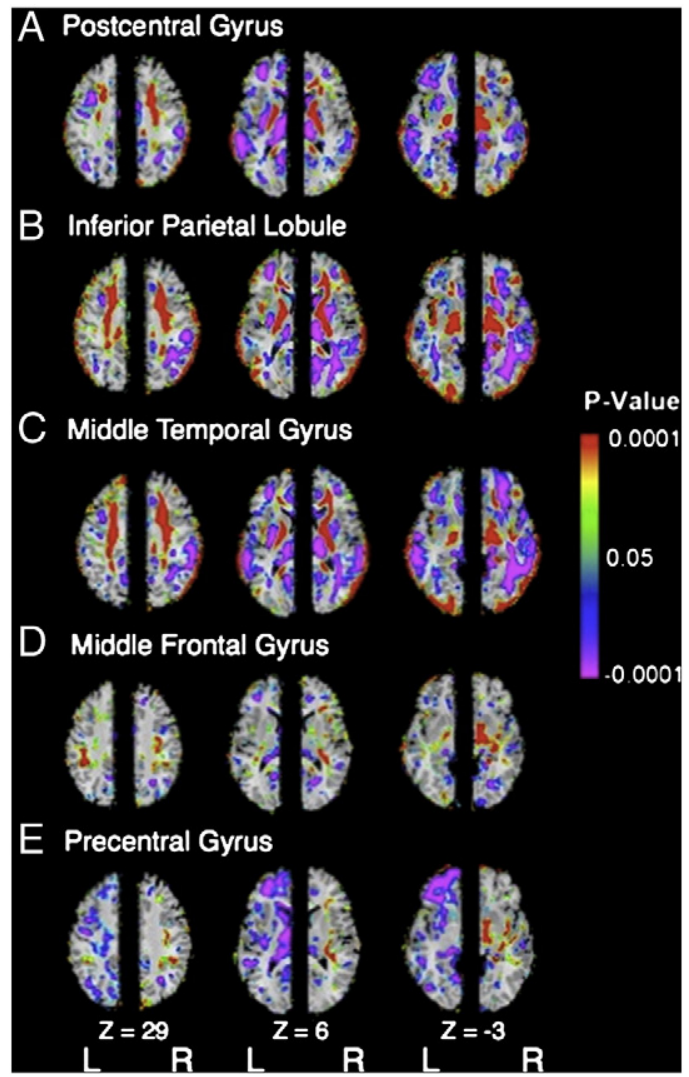

Fig. 3. Correlation of white matter volumes with regional cortical thickness. a. Main effect of average postcentral gyrus cortical thickness on VPW shown at the axial Talairach levels $Z=29,6$ and -3 . Left-right orientation is as in Fig. 1. Right hemisphere shows main effect of average right postcentral gyrus cortical thickness on right hemispheric VPW and left hemisphere shows the main effect of average cortical thickness of the left postcentral gyrus on VPW measures in the left hemisphere. Colors indicate voxels in which this correlation is significant $(p<0.05)$. Regions in red indicate positive correlations, in which white matter hypoplasia co-occurs with cortical thinning and purple indicates inverse correlations. VPW is rescaled for age, sex, and risk group. In the region of significant white matter hypoplasia, which includes the bilateral superior corona radiata, internal capsule, and cerebral peduncle, white matter volume correlates with cortical thickness of the postcentral gyrus. b-e. Main effects of average thickness of the cortical mantle in the (b) inferior parietal lobule, (c) middle temporal gyrus, (d) middle frontal gyrus, and (e) precentral gyrus, on VPW measures. Presentation is as in (a). Cortical thicknesses in the two parietal and temporal areas correlate positively with white matter volumes in the region of significant white matter hypoplasia. Cortical thicknesses of the middle frontal gyrus and precentral gyrus correlate minimally with VPW measures in the region of significant white matter hypoplasia. Crosscorrelations between cortical thickness in the three temporal and parietal cortical areas $(\mathrm{a}-\mathrm{c})$ and between the two frontal cortical areas (d-e) are produced in Table 1. The thicknesses in parietal and temporal areas are highly intercorrelated, accounting for the similarity of effects shown in a-c.

risk than of the severity of current symptoms. Although every effort was made to account for the difference in age between groups, by including age as a covariate, there is the possibility that age may have influenced our results.

We previously identified in the same participants an additional robust marker for MDD that consisted of prominent thinning of the cortical mantle (Peterson et al., 2009) centered over the right lateral surfaces of the frontal, parietal, posterior temporal, and occipital cortices. Cortical thinning throughout these regions correlated with current symptoms of mood and anxiety disorder and current combined symptoms of ADHD. The validity of the marker status of cortical thinning was supported by its presence even in the high-risk participants who have never been ill with MDD or anxiety disorder. The similar findings in the cortical mantle and subcortical white matter prompted us to question whether these markers were related to one another or whether they were relatively independent endophenotypes within our high-risk sample. 


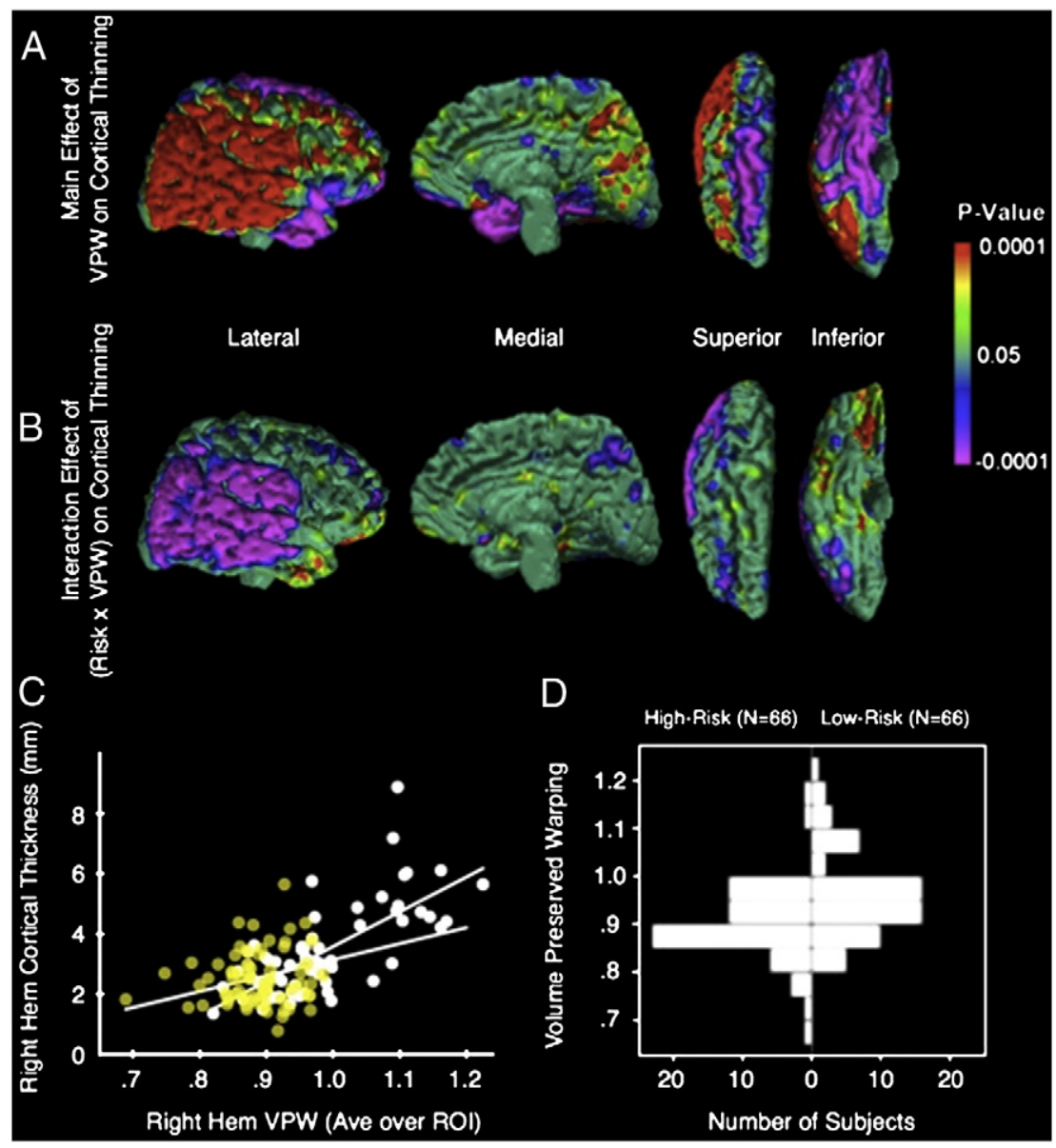

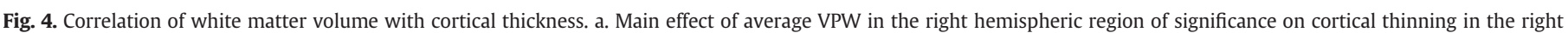

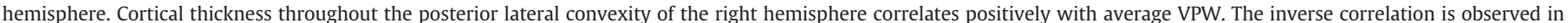

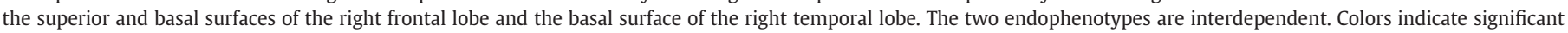

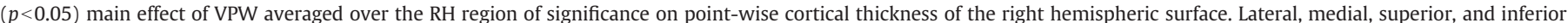

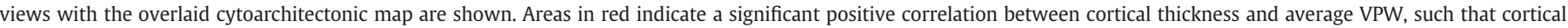

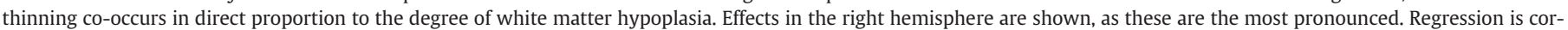

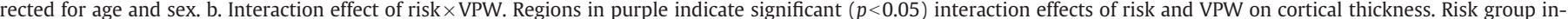

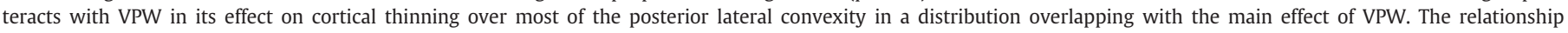

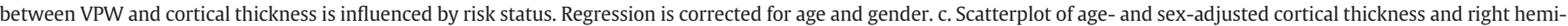

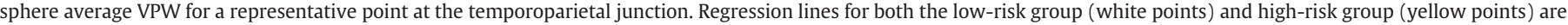

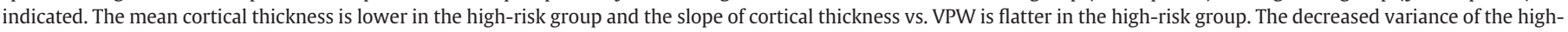

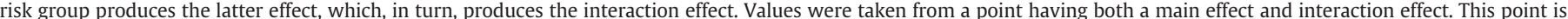

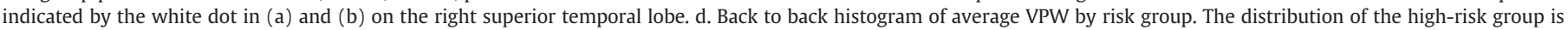

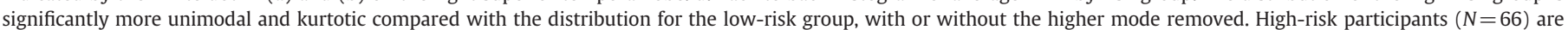

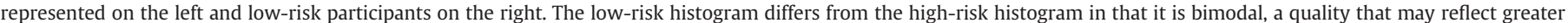
phenotypic heterogeneity.

We assessed the degree of interdependence of these findings by correlating the magnitude of white matter hypoplasia with the magnitude of cortical thinning, and vice versa, in both the high- and lowrisk groups. We found that average cortical thickness with ROIs of the right and left parietal and posterior temporal cortex correlated strongly with the magnitude of white matter hypoplasia in the ipsilateral hemisphere. Moreover, the average magnitude of hypoplasia within white matter regions associated with risk status correlated with cortical thickness throughout the posterior parietal and temporal lobes. Each of these correlations was present in both the highand low-risk groups, suggesting that these white matter regions and posterior cortices covary in the general population, probably because they constitute an interconnected neural circuit. The reduced volumes of the frontal and parietal white matter and the thinning of frontal, posterior temporal, and parietal cortices in the high-risk group are non-independent endophenotypes that represent reduced measures throughout this circuit compared with measures in the low-risk group.
We attempted to identify more precisely the circuits that could be hypoplastic within the high-risk group. Postmortem fiber tracing studies in human and nonhuman primates have shown that the fiber tracts most likely to pass through the regions of frontal and parietal hypoplasia that we identified are the superior longitudinal fasciculus (Petrides and Pandya, 1984; 1988), as well as the corticothalamic, thalamocortical, corticopontine, and corticospinal tracts (Schmahmann and Pandya, 1990; Kunimatsu et al., 2003). Fiber tracking in a separate, unrelated group of 20 healthy young adults demonstrated that the fiber tracts passing through the region where white matter hypoplasia is located are consistent with those found in prior postmortem fiber tracing studies and also include the corpus callosum (Supplemental materials).

Disruption of this group of fiber tracts likely accounts for the significant associations we detected for the magnitude of white matter hypoplasia and the symptoms of MDD, anxiety disorder, and ADHD. The frontoparietal circuit, consisting of the frontal and parietal cortices and their interconnection via the superior longitudinal fasciculus, 
is thought to support top-down attentional processes, spatial biasing (Posner and Petersen, 1990; Peers et al., 2005; Posner and Rothbart, 2009), arousal, and vigilance. Disruption of any one of these functions may compromise self-regulatory capacities and may account for the correlation of local white matter volumes with the severity of ADHD symptoms (Sowell et al., 2003; Cao et al., 2008; Banich et al., 2009). Disruption of the Superior Longitudinal Fasciculus (SLF) and Dorsolateral Prefrontal Cortex (DLPFC) has been observed in Diffusion Tensor Imaging (DTI) studies of MDD (Blood et al., 2010; Wu et al., 2011). Similarly, the frontolimbic circuit, originating in the anterior cingulate, parahippocampal, and entorhinal cortices and projecting through the corona radiata and anterior limb of the internal capsule (ALIC) to the hippocampus, amygdala, hypothalamus, and anterior nucleus of the thalamus, supports the processing of social and emotional stimuli and the regulation of emotions (Peterson et al., 2009; Grossmann et al., 2010). Impaired capacity for self-regulation and emotional-regulation impairs academic and social functioning more generally, thereby compromising work relationships, friendships, and romantic and family relationships in a way that cumulatively impair self-esteem (Duval and Wicklund, 1972; Higgins et al., 1995; Papadakis et al., 2006) and predisposes to MDD (Seligman, 1975). Disruption of the anterior limb of the internal capsule (ALIC) correlated with depression severity in one DTI study of humans (Zhu et al., 2011), while primates reared in adverse conditions had disrupted ALIC on DTI (Coplan et al., 2010). This assertion receives support in our cohort from the strong correlations between scores on the Global Assessment Scale and white matter volumes in the right frontal and parietal lobes.

Identification of this circuit-based endophenotype could guide early diagnosis, treatment, and prevention of MDD. Persons born into families with MDD, for example, could be screened for the presence of this marker. Those who have it could be considered at ultra high-risk for developing MDD and could be offered a preventive intervention (Institute of Medicine (U.S.) Committee on Prevention of Mental Disorders and Substance Abuse among Children, Youth and Young Adults: Research Advances and Promising Interventions, 2009). Moreover, the impairment of attentional and self-regulatory capacities associated with the magnitude of both white matter hypoplasia and right hemisphere cortical thinning, even in those who have not yet become depressed, could suggest new approaches to the treatment of this form of MDD with interventions that are robustly helpful in treating these cognitive and behavioral problems (Raphaelson, 2004; Teicher et al., 2008).

Finally, the identification of this circuit-based endophenotype has important implications for future research. Frontoparietal and frontolimbic circuits should be studied in postmortem and in vivo DTI studies of persons at increased familial risk for MDD. Moreover, those studies should be combined with postmortem imaging studies to establish the histological correlates of our imaging findings, particularly the possible involvement of oligodendroglia (Ongür et al., 1998; Tkachev et al., 2003; Aston et al., 2005) and subcortical axons, which constitute the major cellular components within regions of white matter hypoplasia that we identified. Future research should also aim to determine whether the endophenotype is specific to the familial risk for developing MDD or whether it is also present in those who have MDD with no discernible familial basis. White matter volumes were moderately reduced in low-risk subjects who had a lifetime history of MDD, suggesting that this endophenotype may be present in both familial and non-familial MDD. Family-genetic studies of MDD should incorporate assessment of this endophenotype as a quantitative trait marker to help identify the genetic determinants of MDD and to help reduce the presence of phenocopies that are so detrimental to the identification of linkage. Finally, lesion studies in animal models are needed to determine whether the white matter portion of this putative endophenotype is primary and the cortical portion is secondary, or vice versa, or whether an underlying pathogenic insult could produce hypoplasia in both portions of the circuit simultaneously. The presence of this endophenotype should also help to validate any putative animal model for familial MDD.

\section{Acknowledgments}

This work was supported in part by grants T32-16434, MH36197, and K02-74677 from the National Institute of Mental Health, grants from the National Alliance for Research in Schizophrenia and Affective Disorders (NARSAD), a National Institute on Drug Abuse supplement to grant MH36197, the Sackler Institute at Columbia University, and the Suzanne Crosby Murphy Endowment at Columbia University. We thank J. Sanchez, M. James, and R. Bronen for their technical assistance. None of the authors of the present study has any conflicts of interest. Dr. Myrna Weissman, Dr. Bradley Peterson, and Dr. Marc Dubin had full access to all of the data in the study and take responsibility for the integrity of the data and the accuracy of the data analysis.

\section{Appendix A. Supplementary data}

Supplementary data to this article can be found online at doi:10. 1016/j.pscychresns.2011.11.007.

\section{References}

Amico, F., Meisenzahl, E., Koutsouleris, N., Reiser, M., Moller, H.J., Frodl, T., 2011. Structural MRI correlates for vulnerability and resilience to major depressive disorder. Journal of Psychiatry and Neuroscience 36 (1), 15-22.

Aston, C., Jiang, L., Sokolov, B.P., 2005. Transcriptional profiling reveals evidence for signaling and oligodendroglial abnormalities in the temporal cortex from patients with major depressive disorder. Molecular Psychiatry 10, 309-322.

Banich, M.T., Burgess, G.C., Depue, B.E., Ruzic, L., Bidwell, L.C., Hitt-Laustsen, S., Du, Y.P., Willcutt, E.G., 2009. The neural basis of sustained and transient attentional control in young adults with ADHD. Neuropsychologia 47, 3095-3104.

Beidel, D.C., Turner, S.M., 1997. At risk for anxiety: I. Psychopathology in the offspring of anxious parents. Journal of the American Academy of Child and Adolescent Psychiatry 36, 918-924

Benjamini, Y., Hochberg, Y., 1995. Controlling the false discovery rate: a practical and powerful approach to multiple testing. Journal of the Royal Statistical Society, Series B 57 (1), 289-300.

Blood, A.J., Iosifescu, D.V., Makris, N., Perlis, R.H., Kennedy, D.N., Dougherty, D.D., Kim, B.W., Lee, M.J., Wu, S., Lee, S., Calhoun, J., Hodge, S.M., Fava, M., Rosen, B.R. Smoller, J.W., Gasic, G.P., Breiter, H.C., Phenotype Genotype Project on Addiction and Mood Disorders, 2010. Microstructural abnormalities in subcortical reward circuitry of subjects with major depressive disorder. PLoS One 5 (11).

Cao, Q., Zang, Y., Zhu, C., Cao, X., Sun, L., Zhou, X., Wang, Y., 2008. Alerting deficits in children with attention deficit/hyperactivity disorder: event-related fMRI evidence. Brain Research 1219, 159-168.

Cook, I.A., Hunter, A.M., Abrams, M., Siegman, B., Leuchter, A.F., 2009. Midline and right frontal brain function as a physiologic biomarker of remission in major depression. Psychiatry Research: Neuroimaging 174, 152-157.

Coplan, J.D., Abdallah, C.G., Tang, C.Y., Mathew, S.J., Martinez, J., Hof, P.R., Smith, E.L.P., Dwork, A.J., Perera, T.D., Pantol, G., Carpenter, D., Rosenblum, L.A., Shungu, D.C., Gelernter, J., Kaffman, A., Jackowski, A., Kaufman, J., Gorman, J.M., 2010. The role of early life stress in development of the anterior limb of the internal capsule in nonhuman primates. Neuroscience Letters 480 (2), 93-96.

Dinov, I.D., Mega, M.S., Thompson, P.M., Lee, L., Woods, R.P., Holmes, C.J., Sumners, D.W., Toga, A.W., 2000. Analyzing functional brain images in a probabilistic atlas: a validation of subvolume thresholding. Journal of Computer Assisted Tomography 24, 128-138.

Drevets, W.C., Price, J.L., Simpson, J.R., Todd, R.D., Reich, T., Vannier, M., Raichle, M.E., 1997. Subgenual prefrontal cortex abnormalities in mood disorders. Nature 386, 824-827.

Duval, S., Wicklund, R.A., 1972. A Theory of Objective Self Awareness. Academic Press, Oxford.

Faraone, S.V., Biederman, J., 1997. Do attention deficit hyperactivity disorder and major depression share familial risk factors? The Journal of Nervous and Mental Disease $185,533-541$

Frodl, T.S., Koutsouleris, N., Bottlender, R., Born, C., Jager, M., Scupin, I., Reiser, M. Moller, H.J., Meisenzahl, E.M., 2008. Depression-related variation in brain morphology over 3 years: effects of stress? Archives of General Psychiatry 65, 1156-1165.

Frodl, T., Reinhold, E., Koutsouleris, N., Reiser, M., Meisenzahl, E.M., 2010. Interaction of childhood stress with hippocampus and prefrontal cortex volume reduction in major depression. Journal of Psychiatric Research 44 (13), 799-807.

Gershon, E.S., 1983. Genetics of the major psychoses. Research Publications - Association for Research in Nervous and Mental Disease 60, 121-144 (Review).

Gottesman, I.I., Gould, T.D., 2003. The endophenotype concept in psychiatry: etymology and strategic intentions. The American Journal of Psychiatry 160, 636-645.

Grossmann, T., Oberecker, R., Koch, S.P., Friederici, A.D., 2010. The developmental origins of voice processing in the human brain. Neuron $65,852-858$ 
Higgins, E.T., Loeb, I., Moretti, M., 1995. Self-discrepancies and developmental shifts in vulnerability: life transitions in the regulatory significance of others. In: Cicchetti, D. Toth, S.L. (Eds.), Emotion, Cognition and Representation. University of Rochester Press, Rochester, N.Y., pp. 191-230.

Hill, J., Pickles, A., Rollinson, L., Davies, R., Byatt, M., 2004. Juvenile-versus adult-onset depression: multiple differences imply different pathways. Psychological Medicine 34 (08), 1483-1493.

Institute of Medicine (U.S.) Committee on Prevention of Mental Disorders and Substance Abuse among Children, Youth and Young Adults: Research Advances and Promising Interventions, 2009. Preventing Mental, Emotional, and Behavioral Disorders among Young People: Progress and Possibilities. National Academies Press, Washington, D.C.

Jorge, R.E., Robinson, R.G., Moser, D., Tateno, A., Crespo-Facorro, B., Arndt, S., 2004 Major depression following traumatic brain injury. Archives of General Psychiatry $61,42-50$.

Kunimatsu, A., Aoki, S., Masutani, Y., Abe, O., Mori, H., Ohtomo, K., 2003. Three-dimensiona white matter tractography by diffusion tensor imaging in ischaemic stroke involving the corticospinal tract. Neuroradiology 45, 532-535.

Mazziotta, J., Toga, A., Evans, A., Fox, P., Lancaster, J., Zilles, K., Woods, R., Paus, T., Simpson, G., Pike, B., Holmes, C., Collins, L., Thompson, P., MacDonald, D. Iacoboni, M., Schormann, T., Amunts, K., Palomero-Gallagher, N., Geyer, S., Parsons, L., Narr, K., Kabani, N., Le Goualher, G., Boomsma, D., Cannon, T., Kawashima, R., Mazoyer, B., 2001. A probabilistic atlas and reference system for the human brain: International Consortium for Brain Mapping (ICBM). Philosophical Transactions of the Royal Society of London. Series B, Biological Sciences 356, 1293-1322.

McGuffin, P., 1984. Biological markers and psychosis. Psychological Medicine 14 (2), 255-258.

Mendlewicz, J., Charles, G., Franckson, J.M., 1982. The dexamethasone suppression test in affective disorder: relationship to clinical and genetic subgroups. The British Journal of Psychiatry 141, 464-470.

Ongür, D., Drevets, W.C., Price, J.L., 1998. Glial reduction in the subgenual prefronta cortex in mood disorders. Proceedings of the National Academy of Sciences of the United States of America 95, 13290-13295.

Papadakis, A.A., Prince, R.P., Jones, N.P., Strauman, T.J., 2006. Self-regulation, rumination, and vulnerability to depression in adolescent girls. Development and Psychopathology $18,815-829$.

Peers, P.V., Ludwig, C.J.H., Rorden, C., Cusack, R., Bonfiglioli, C., Bundesen, C., Driver, J. Antoun, N., Duncan, J., 2005. Attentional functions of parietal and frontal cortex. Cerebral Cortex 15, 1469-1484.

Peterson, B.S., Warner, V., Bansal, R., Zhu, H., Hao, X., Liu, J., Durkin, K., Adams, P.B. Wickramaratne, P., Weissman, M.M., 2009. Cortical thinning in persons at increased familial risk for major depression. Proceedings of the National Academy of Sciences of the United States of America 106, 6273-6278.

Petrides, M., Pandya, D.N., 1984. Projections to the frontal cortex from the posterior parietal region in the rhesus monkey. The Journal of Comparative Neurology 228, 105-116.

Petrides, M., Pandya, D.N., 1988. Association fiber pathways to the frontal cortex from the superior temporal region in the rhesus monkey. The Journal of Comparative Neurology 273, 52-66.

Posner, M.I., Petersen, S.E., 1990. The attention system of the human brain. Annual Review of Neuroscience $13,25-42$.
Posner, M.I., Rothbart, M.K., 2009. Toward a physical basis of attention and self regulation. Physics of Life Reviews 6, 103-120.

Price, J., 1968. Neurotic and endogenous depression: a phylogenetic view. The British Journal of Psychiatry 114 (506), 119-120.

Raphaelson, M., 2004. Stimulants and attention-deficit/hyperactivity disorder. JAMA : The Journal of the American Medical Association 292, 2214 author reply 2214.

Robinson, R.G., Price, T.R., 1982. Post-stroke depressive disorders: a follow-up study of 103 patients. Stroke 13, 635-641.

Schmahmann, J.D., Pandya, D.N., 1990. Anatomical investigation of projections from thalamus to posterior parietal cortex in the rhesus monkey: a WGA-HRP and fluorescent tracer study. The Journal of Comparative Neurology 295, 299-326.

Seligman, M., 1975. Helplessness: On Depression, Development, and Death. Freeman, San Francisco.

Seminowicz, D.A., Mayberg, H.S., McIntosh, A.R., Goldapple, K., Kennedy, S., Segal, Z., Rafi-Tari, S., 2004. Limbic-frontal circuitry in major depression: a path modeling metanalysis. NeuroImage 22, 409-418.

Sled, J.G., Zijdenbos, A.P., Evans, A.C., 1998. A nonparametric method for automatic correction of intensity nonuniformity in MRI data. IEEE Transactions on Medical Imaging 17, 87-97.

Smith, S.M., 2002. Fast robust automated brain extraction. Human Brain Mapping 17, 143-155.

Sowell, E R. Thompson, P.M., Welcome, S.E. Henkenius, A.L, Toga, A.W Peterson, B.S. 2003. Cortical abnormalities in children and adolescents with attention-deficit hyperactivity disorder. Lancet 362, 1699-1707.

Teicher, M.H., Polcari, A., McGreenery, C.E., 2008. Utility of objective measures of activity and attention in the assessment of therapeutic response to stimulants in children with attention-deficit/hyperactivity disorder. Journal of Child and Adolescent Psychopharmacology 18, 265-270.

Tkachev, D., Mimmack, M.L., Ryan, M.M., Wayland, M., Freeman, T., Jones, P.B., Starkey, M., Webster, M.J., Yolken, R.H., Bahn, S., 2003. Oligodendrocyte dysfunction in schizophrenia and bipolar disorder. Lancet 362, 798-805.

Tremblay, L.K., Naranjo, C.A., Graham, S.J., Herrmann, N., Mayberg, H.S., Hevenor, S., Busto, U.E., 2005. Functional neuroanatomical substrates of altered reward processing in major depressive disorder revealed by a dopaminergic probe. Archives of General Psychiatry 62, 1228-1236.

Weissman, M.M., Wickramaratne, P., Nomura, Y., Warner, V., Verdeli, H., Pilowsky, D.J. Grillon, C., Bruder, G., 2005. Families at high and low risk for depression: a 3-generation study. Archives of General Psychiatry 62, 29-36.

Wickramaratne, P.J., Weissman, M.M., 1998. Onset of psychopathology in offspring by developmental phase and parental depression. Journal of the American Academy of Child and Adolescent Psychiatry 37, 933-942.

Wu, F., Tang, Y., Xu, K., Kong, L., Sun, W., Wang, F., Kong, D., Li, Y., Liu, Y., 2011. Whiter matter abnormalities in medication-naive subjects with a single short-duration episode of major depressive disorder. Psychiatry Research 191 (1), 80-83.

Xu, D., Hao, X., Bansal, R., Plessen, K., Geng, W., Hugdahl, K., Peterson, B., 2007. Unifying the analyses of anatomical and diffusion tensor images using volume-preserved warping. Journal of Magnetic Resonance Imaging 25, 612.

Zhu, X., Wang, X., Xiao, J., Zhong, M., Liao, J., Yao, S., 2011. Altered white matter integrity in first-episode, treatment-naive young adults with major depressive disorder: a tract-based spatial statistics study. Brain Research 1369, 223-229. 